\title{
Causal modeling in a multi-omic setting: insights from GAW20
}

\author{
Jonathan Auerbach ${ }^{1 \dagger}$, Richard Howey ${ }^{2 \dagger}$, Lai Jiang ${ }^{3 \dagger}$, Anne Justice ${ }^{4 \dagger}$, Liming Li ${ }^{5 \dagger}$, Karim Oualkacha ${ }^{6 \dagger}$, \\ Sergi Sayols-Baixeras ${ }^{7+}$ and Stella W. Aslibekyan ${ }^{8^{*}}$ \\ From Genetic Analysis Workshop 20 \\ San Diego, CA, USA. 4-8 March 2017
}

\begin{abstract}
Background: Increasingly available multilayered omics data on large populations has opened exciting analytic opportunities and posed unique challenges to robust estimation of causal effects in the setting of complex disease phenotypes. The GAW20 Causal Modeling Working Group has applied complementary approaches (eg, Mendelian randomization, structural equations modeling, Bayesian networks) to discover novel causal effects of genomic and epigenomic variation on lipid phenotypes, as well as to validate prior findings from observational studies.

Results: Two Mendelian randomization studies have applied novel approaches to instrumental variable selection in methylation data, identifying bidirectional causal effects of CPT1A and triglycerides, as well as of RNMT and C6orf42, on high-density lipoprotein cholesterol response to fenofibrate. The CPT1A finding also emerged in a Bayesian network study. The Mendelian randomization studies have implemented both existing and novel steps to account for pleiotropic effects, which were independently detected in the GAW20 data via a structural equation modeling approach. Two studies estimated indirect effects of genomic variation (via DNA methylation and/or correlated phenotypes) on lipid outcomes of interest. Finally, a novel weighted $R^{2}$ measure was proposed to complement other causal inference efforts by controlling for the influence of outlying observations.
\end{abstract}

Conclusions: The GAW20 contributions illustrate the diversity of possible approaches to causal inference in the multi-omic context, highlighting the promises and assumptions of each method and the benefits of integrating both across methods and across omics layers for the most robust and comprehensive insights into disease processes.

Keywords: Genomics, Epigenomics, Causal inference, Mendelian randomization, Bayesian networks, Structural equation modeling, Outliers, Variable selection methods

\section{Background}

The question of causality-and the distinction between association and causation-lies at the heart of scientific inquiry. In genomic research specifically, the principal focus is on estimating causal effects of DNA sequence variants on downstream phenotypes, ideally revealing the underlying biological mechanisms and identifying

\footnotetext{
* Correspondence: saslibek@uab.edu

${ }^{\dagger} J$ Conathan Auerbach, Richard Howey, Lai Jiang, Anne Justice, Liming Li, Karim Oualkacha and Sergi Sayols-Baixeras contributed equally to this work.

${ }^{8}$ Department of Epidemiology, University of Alabama at Birmingham, 1665

University Blvd, RPHB 230J, Birmingham, AL 35294, USA

Full list of author information is available at the end of the article
}

novel treatment targets along the way. At first glance, the task of causal inference in genomics seems trivial: individual genotype is inherited following Mendel's laws of segregation and independent assortment, and stays stable after conception, obviating such concerns as reverse causation, confounding (except for population stratification [1]), or other spurious relationships with the disease phenotype. Yet most genome-wide association studies (GWAS) to date have failed to produce solid evidence of causality, and most statistically significant GWAS findings are often not replicated in other populations [2] or functionally corroborated by experimental models. The reasons for this failure to progress from association to causation are manifold, and

(c) The Author(s). 2018 Open Access This article is distributed under the terms of the Creative Commons Attribution 4.0 International License (http://creativecommons.org/licenses/by/4.0/), which permits unrestricted use, distribution, and 
include poor study design and analysis practices, the "winner's curse [3]," low frequency of disease-causing alleles, inadequate representation of diverse populations, epistatic effects, gene-environment interactions, and various logistical and financial hurdles to conducting rigorous follow-up investigations. Largely because of these limitations, few genomic findings to date have resulted in translational breakthroughs.

The challenge of causal inference is even more daunting in methylome-wide association studies (MWAS), which interrogate associations between variation in DNA methylation and disease phenotypes. Although MWAS findings suffer from some of the same pitfalls as GWAS (eg the "winner's curse"), they are additionally vulnerable to reverse causation and effects of confounding variables, as most of the epigenome is not heritable [4] and can be influenced by aging, disease, and a variety of environmental exposures [5]. Furthermore, single-nucleotide polymorphisms (SNPs) located in the vicinity of cytosine-phosphate-guanine (CpG) sites, which account for most of the methylation variance in the mammalian genome, have robust effects on the methylation status of the neighboring (cis-) CpG sites [6], potentially confounding the observed epigenomic associations with the phenotype.

The GAW20 provided an opportunity to infer causal relations between the genotype, longitudinal measures of DNA methylation, and metabolic phenotypes, collected in a large family study of lipid-lowering treatment with fenofibrate [7]. In this manuscript, we describe the methodological approaches taken by members of the GAW20 Causal Modeling Working Group, present the overarching themes and insights, and situate GAW20 findings within the broader context of genetic epidemiology research.

\section{Methods}

The characteristics of the GAW20 data set, built from the Genetics of Lipid Lowering Drugs and Diet Network (GOLDN) study, are described in detail by Aslibekyan and colleagues [7]. Briefly, the data included epigenome-wide DNA methylation as ascertained by the Illumina Infinium Human Methylation $450 \mathrm{~K}$ array before and after a 3-week treatment with fenofibrate, 718,542 SNPs genotyped by the Affymetrix 6.0 array, phenotypes (plasma lipids and metabolic syndrome), and relevant covariates on 1105 individuals from 188 families. In addition to the real data from GOLDN participants, the GAW20 release included 200 replicates of simulated posttreatment methylation and phenotype (namely triglycerides) measurements, which were described by Province and colleagues [8].

Table 1 summarizes the tools and techniques employed by the 6 research teams participating in the GAW20 Causal Modeling Working Group discussion. All teams analyzed the real data, and Howey and associates [9] additionally tested their method on the simulated measurements with prior knowledge of the "answers" (ie, causal variants). Furthermore, both Jiang and associates [10] and Li and colleagues [11] conducted their own simulations to compare the performance of their methods to their predecessors. Taking advantage of the unique multi-omic context of GAW20, all teams integrated both SNP and CpG methylation data, except for Auerbach and associates, (Auerbach J, Hsu Y, Zhou W, Lo SH: Resistant R-squared for summarizing genetic effects. In preparation) who restricted their analysis to the DNA sequence variation. The team approaches represented a combination of agnostic (exploratory), genome-wide tests [11] and validation of prior reported associations (confirmatory) by using, for example, Mendelian randomization (MR) [10, 12], causal networks [9], structural equations modeling (SEM [13]), or a novel $R^{2}$ measure that moderates the influence of outliers. Although most teams operated within established methodologic paradigms (eg, MR or SEM) $[9,10,12,13]$, Auerbach and associates and Li and colleagues [11] created new tools and tested their performance in the GAW20 data. On balance, the 6 teams varied considerably in their perspectives, approaches, and results, as discussed in further detail in the following sections.

\section{Results \\ Motivation}

The contributions from the Causal Modeling Working Group reflected considerable conceptual diversity. Broadly

Table 1 Summary of statistical methods used by the GAW20 Causal Modeling Group

\begin{tabular}{|c|c|c|c|c|c|c|}
\hline & Auerbach $\left(R^{2}\right)$ & Howey (Bayesian networks) & Jiang (MR) & Justice (SEM) & $\begin{array}{l}\text { Li (Mechanistic } \\
\text { modeling) }\end{array}$ & Sayols-Baixeras (MR) \\
\hline Adjustment for family relatedness & $x$ & & $x$ & $x$ & $x$ & $x$ \\
\hline Bootstrapping & & $x$ & $x$ & & & \\
\hline Mendelian randomization & & & $x$ & & & $x$ \\
\hline Principal components & & $x$ & $x$ & $x$ & & \\
\hline Causal networks & & $x$ & & $x$ & & \\
\hline Imputed SNPs & & & $x$ & & & \\
\hline Optimization & & $x$ & $x$ & & & \\
\hline
\end{tabular}


speaking, the teams aimed to strengthen the inferences that arise from observational studies reporting associations between DNA sequence or methylation variation and lipid phenotypes. In the case of Auerbach and associates, who only interrogated the genetic contribution to the phenotype, the team aimed to develop a novel $\mathrm{R}^{2}$ measure that would be robust to outliers. The remaining 5 studies also considered effects of epigenomic variation, which was the primary focus of the $2 \mathrm{MR}$ studies $[10,12]$ that used genotype as the instrumental variable for methylation, phenotype, or both. In contrast to using genotype as a mere instrument, the studies by $\mathrm{Li}$ and colleagues [11] and Justice and associates [13] focused on sequence variation as the exposure, testing whether the total effect of the SNP on the phenotype also includes indirect effects mediated by neighboring $\mathrm{CpG}$ methylation or correlated lipid phenotypes. Finally, Howey and associates [9] sought to identify possible causal relationships between and within both omic layers and the phenotypes with the use of Bayesian networks. Overall, the GAW20 experience highlighted the utility of integrating across types of omic data to (a) aid causal inference and (b) paint a more complete and accurate picture of human lipid variation.

\section{Defining causality in GAW20}

Historically, causality has been defined under 1 of 2 main frameworks, commonly referred to by their most distinctive features: potential outcomes [14] and directed graphs [15]. Both paradigms were represented among the 6 GAW20 research teams. The potential outcomes framework treats randomized controlled experiments as the gold standard for estimating causal relationships. Randomization avoids complications that occur when the manner in which subjects are assigned a treatment (or subject to an exposure) accounts for the differences in outcomes in addition to the treatment (or exposure) itself. That is not to say randomization is a statistical panacea; Auerbach and associates showed how causal estimates may be sensitive to selection effects even when treatments are randomized. Nevertheless, randomization eliminates many of the sources of confounding that could create spurious relationships and biased effect estimation. The MR approaches implemented by Jiang and colleagues [10] and Sayols-Baixeras and associates [12] represent an extension of this framework to quasiexperimental design via instrumental variable analysis [16].

In contrast, the directed graphs framework relies on deterministic laws of science for describing causal relationships. It follows that complete knowledge of the underlying mechanism of a phenomenon reveals any cause and effect relationships. In practice, it is often impossible to account for every possible relationship that might exist between a set of variables. Directed graphs take advantage of probability distributions and sequential events in time or space to simplify characterization of the data generating process. $\mathrm{Li}$ and associates [11] used mechanistic modeling to explain how genetic factors influence phenotype, taking advantage of the fact that genetic factors precede the phenotype. Use of structural equations to describe an outcome, represented in GAW20 by Justice and associates [13], has its origins in path diagrams [17]. The power of the directed graph framework lies in its ability to depict complicated relationships. In GAW20, Howey and associates [9] represented this framework with a Bayesian network, which consists of a directed acyclic graph and a set of parameters in all conditional probability distributions. Although this procedure is computationally expensive, it allows simultaneous analytic consideration of a large number of possible mechanisms.

Even though the interpretations of causality varied within the Causal Modeling Working Group, the teams came to the consensus definition of causal inference as the process that evaluates (and potentially rules out) competing explanations for observed associations between exposures (eg, genomic variation) and outcomes (eg, metabolic phenotypes). All analyses took place in the multi-omic setting of GAW20 data, which held several causal possibilities as summarized in Fig. 1, including confounding and reverse causation scenarios. Additionally, directed acyclic graphs (Fig. 1) can be expanded to accommodate pleiotropic effects considered by multiple GAW20 analyses [10, 12, 13]. Similarly, these graphs (Fig. 1a-h) can be modified to include repeated measurements of both methylation and phenotypic data, adding fenofibrate treatment and/or baseline lipid concentrations as potential nodes. However, even though several teams used multiple lipid measurements in their analyses, longitudinal dynamics were not a major focus of the Causal Modeling Working Group. For example, no Causal Modeling Working Group team interrogated changes in epigenetic patterns over the treatment period, likely because of the inextricable confounding between fenofibrate and batch effects on methylation measurements, described in detail elsewhere [7]. Beyond GAW20, the question of temporal variation in epigenetic effects remains similarly unexplored, but an increasing number of large-scale cohorts are currently in process of obtaining serial methylation data, promising future opportunities for adapting current causal inference methods to longitudinal epigenetics.

\section{Theoretical and practical challenges \\ Data}

The first set of challenges for the Causal Modeling Working Group was presented by the structure of the GAW20 data set. The moderate sample size $(N=1105)$, particularly by the standards of MR analysis, hampered detection of statistically significant effects. An important 


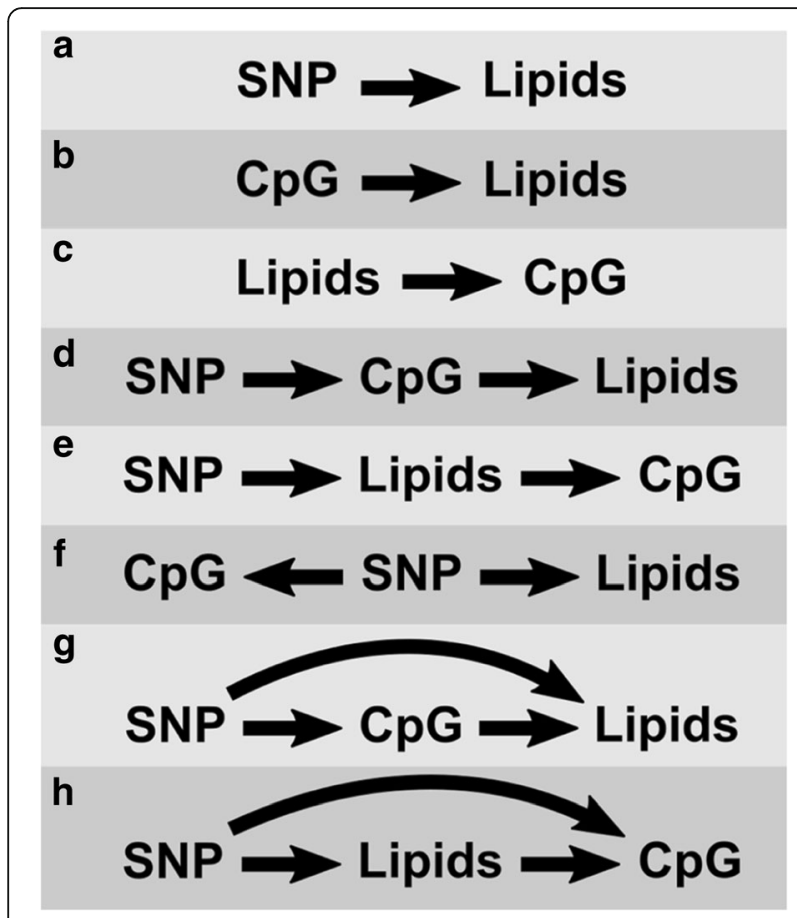

Fig. 1 Possible (nonexhaustive) causal structures of the GAW20 data. Directed acyclic graphs illustrating several causal scenarios possible in the GAW20 data: (a) and (b) represent direct effects of DNA sequence variation and DNA methylation on lipids, respectively; (c) represents a direct effect of lipids on DNA methylation; (d) and (e) represent full mediation scenarios, in which the effect of DNA sequence variation on lipids/DNA methylation occurs solely through DNA methylation/lipids, respectively; (f) shows confounding of the DNA methylation-lipids relationship by DNA sequence variation; (g) and (h) depict partial mediation scenarios, in which DNA sequence variation affects lipids/DNA methylation both directly and through DNA methylation/lipids, respectively

step that all teams performed, but that can be often overlooked, was ensuring that the data was suitable for analysis through formatting and cleaning, such as checking Hardy-Weinberg equilibrium and/or minor allele frequencies and handling missing data. All teams adjusted for covariates (eg, age and sex) in their analyses to address bias resulting from confounding or the potential mediating effects of such variables. A special case of covariate adjustment necessary in GOLDN/GAW20 data is accounting for family relatedness, which is essential for producing valid estimates of effect in genetic studies. That was accomplished by implementing existing methods accounting for family structure [10-13], extending such methods, or not accounting for family structure while observing this limitation [9]. Potential technical artifacts in the DNA methylation data were addressed by including principal components in MWAS analyses $[9,10,13]$. Finally, as outlying observations can threaten the accuracy of estimating average effects, Auerbach and associates derived a weighted $R^{2}$ measure that was resistant to such influences and could be used to strengthen inference from traditional statistics.

\section{Analytic assumptions}

The validity of causal effects estimated by all statistical methods hinges on satisfying the underlying assumptions, which are not always empirically testable. For example, MR estimators must meet the general assumptions for any instrumental variable, which include a robust association with the risk factor (testable), no common causes between the genotype and the phenotype of interest (not testable, but usually satisfied by random assortment of alleles-with the exception of population stratification), and no pleiotropic effects (ie, the genetic instrumental variable must only be associated with the phenotype of interest through the intermediate phenotype that is it meant to represent; not directly testable). To address the third assumption, Sayols-Baixeras and colleagues [12] used the widely accepted MR-Egger method [18] to rule out pleiotropy. In contrast, Jiang and associates [10] developed a novel method (constrained instrumental variables) that adaptively selects the optimal subset of instrumental variables that maximize associations with the intermediate phenotype of interest while accounting for potential pleiotropic effects. The constrained instrumental variables findings were comparable, albeit not identical, to MR-Egger and two-stage least-squares MR, identifying 2 additional causal associations as well as the 1 association detected by established methods. Meanwhile, Justice and associates [13] justified the concern about pleiotropic effects in the GAW20 data, reporting independent direct effects of rs 964184 on both triglycerides and high-density lipoprotein cholesterol, and thus indicating existence of true pleiotropy.

Similar problems exist in Bayesian network analyses [9], requiring that suitable data are included in the analysis to anchor the correct direction of the causal relationships between variables of interest. Other assumptions behind Bayesian networks include acyclic relationships between variables; multinomial distribution of discrete variables and normal distribution of continuous variables; independence between variables conditional on their parents; and no parents for SNP variables. Of those, the normality assumption is the most problematic as genomic variation is coded as $0 / 1 / 2$, but is still modeled continuously to avoid problems posed by low minor allele frequencies.

The SEM analysis by Justice and colleagues [13] assumes that all data are missing at random, which is especially unlikely in longitudinal data. Justice and associates [13] found no association between missingness and any informative variable in the data set (eg, sex, age, metabolic syndrome status). However, the GAW20 data set does not contain all potentially relevant confounders that may be 
predictive of missingness; consequently, the missing at random assumption may not be valid.

The likelihood inference proposal for indirect estimation (LIPID) developed by $\mathrm{Li}$ and colleagues [11] focuses on the CpG sites that are regulated by neighboring DNA sequence variants (methylation quantitative trait loci), and also have a causal effect on the phenotype. However, current estimates indicate methylation quantitative trait loci regulation at $<40 \%$ of CpG sites [19], limiting the applicability of LIPID in studies of DNA methylation. Additionally, prior MR studies of lipids [20] demonstrated effects of the phenotype on CpG methylation rather than vice versa (ie, in the direction assumed by LIPID). Although the GAW20 findings are consistent with either direction of effect (methylation $\rightarrow$ lipids and lipids $\rightarrow$ methylation) $[9,12]$, only one direction satisfies the analytic assumption of the LIPID method.

Furthermore, both $\mathrm{Li}$ and colleagues [11] and Auerbach and colleagues adjusted for familial relatedness in the GAW20 data using the theoretical kinship matrix, which assumes that the founder populations are completely unrelated (which is unlikely in the context of human population history [21], particularly in close-knit communities of Utah and Minnesota that served as the study base for GOLDN/GAW20) as well as correctly specified. These issues could be obviated by estimating kinship based on SNP data rather than self-reported pedigree information [21]. The methods implemented by $\mathrm{Li}$ and associates [11] and Auerbach and associates also assume independence of study participants conditional on their genotype. Because environmental variables within a household are likely to be correlated, this assumption likely does not hold, and merits further investigation with a fuller data set that includes such factors as diet, lifestyle, and other potential nongenetic effects.

Finally, as all teams used linear regression models, all methods used in the Causal Modeling Working Group are based on the standard assumptions of error independence, homoscedasticity, and multivariate normality, as well as a linear relationship between the genetic/epigenetic variants and phenotypes that is unlikely to completely capture the underlying biologic complexity.

\section{Subjective choices}

Related to the issue of methodologic assumptions, many of the analyses used by teams required some form of subjective choices, such as weighted covariance matrix, the size of methylation probe sets [10] or SNP windows $[10,12]$, Bayesian network variables [9], and imputation parameters [10]. Future studies are warranted to examine the sensitivity of the proposed methods to such arbitrary initial conditions.

\section{Computation}

All analyses performed by the Causal Modeling Working Group faced a number of computation challenges, included but not limited to bootstrapping [9, 10], imputation [10], optimization algorithms [9], and parallelization of analyses (all teams).

\section{Discussion}

The multilayered data environment provided by GAW20 was suitable for numerous avenues of causal inquiry, spurring vastly different approaches that unsurprisingly produced different results. One exception to that pattern was the effect of triglycerides on methylation loci in CPT1A, captured by both MR (in GAW20 and elsewhere [20]) and Bayesian networks, with the latter estimating the likelihood of such a causal association at $58 \%$ [9]. Notably, in addition to replicating the effect of lipids on CPT1A methylation, Sayols-Baixeras and associates [12] also showed that the reverse effect (ie, from methylation to triglycerides) cannot be ruled out. This is consistent with the estimate from the Bayesian network analysis, in which probability values near $50 \%$ indicate that the causal relationship is equally likely to be in either direction.

The convergence of the 2 distinct approaches on the same finding showed that the potential outcomes and the directed acyclic graph paradigms are not irreconcilable. Indeed, researchers have identified a variety of conditions necessary to bridge the 2 frameworks $[15,22$, 23]. However, as a consequence of the complexity of causal inference, a variety of strategies are still useful for identifying causal relationships. In GAW20, the investigation of the CPT1A methylation $\rightarrow$ triglyceride relationship was hindered by the lack of a strong genetic instrument for the methylation loci. However, future applications of novel variable selection methods such as constrained instrumental variables [10] may be able to provide strong instruments for bidirectional MR analyses to further interrogate this epigenetic finding. Conversely, findings of causal effects of methylation in RNMT and C6orf42 on high-density lipoprotein cholesterol response to fenofibrate reported by Jiang and associates [10] were subsequently bidirectionally reanalyzed to rule out reverse causality; the resulting evidence did not support a causal effect of high-density lipoprotein changes on DNA methylation. Another impediment to considering all possible causal pathways was posed by the insufficient sample size. For example, Justice and associates [13] only modeled the mediating effect of methylation on the pathway between SNP and triglyceride, because a SEM analysis examining a larger number of possible scenarios-including one where lipids act as mediators of the SNP-methylation relationship-would have been underpowered in the GAW20 data set. 
Although most analyses undertook a candidate gene approach, aiming to strengthen causal inference for already known loci, $\mathrm{Li}$ and associates [11] used their LIPID method to identify novel genes implicated in lipid metabolism. Of 13,968 considered genes, they identified FAT1 and DCTN6 as having a significant effect on triglyceride phenotypes. These 2 genes were not among those examined in the other analyses of the Causal Modeling Working Group, which precludes direct comparisons with other integrative methods, such as with the structural equations models implemented by Justice and colleagues [13]. However, both FAT1 and DCTN6 are biologically plausible, and are annotated to lipid metabolism in the Database for Annotation, Visualization, and Integrated Discovery database [11]. Furthermore, in a rat model of in utero undernutrition followed by leptin treatment, Dctn6 emerged as a target for "thrifty" metabolic programming [24], demonstrating its epigenetic connection to metabolic phenotypes. Given the computational efficiency of the LIPID method and its demonstrated superior statistical power compared to existing methods [11], these findings illustrate the promise of this approach for gene discovery in future integrative analyses of SNP/CpG methylation data.

\section{Conclusions}

The experience of the GAW20 Causal Modeling Working Group illustrated several challenges and promises of causal inference in the multi-omic data environment. Employing diverse strategies to identify novel causal genes or validate prior observational findings, GAW20 contributions showed that there is no statistical "silver bullet," as each method is predicated on its own-often not directly verifiable-assumptions. Because true causal findings are likely to be detected by more than one algorithm, future causal inference approaches can benefit from integrating multiple methods with complementary strengths and limitations. Moreover, as the availability of omics data on large population increases exponentially, it is imperative that future efforts continue exploring novel ways of leveraging all available omics layers for robust causal inference, as well as increasingly more accurate modeling of complex trait etiology.

\section{Abbreviations}

CpG: Cytosine-phosphate-guanine; DNA: Deoxyribonucleic acid: GAW20: Genetic Analysis Workshop 20; GOLDN: Genetics of Lipid Lowering Drugs and Diet Network; GWAS: genome-wide association study; LIPID: Likelihood inference proposal for indirect estimation; MR: Mendelian randomization; MWAS: Methylome-wide association study; SEM: Structural equation modeling; SNP: Single nucleotide polymorphism

\section{Funding}

Publication of the proceedings of Genetic Analysis Workshop 20 was supported by National Institutes of Health grant R01 GM031575. The Genetic Analysis Workshops are supported by National Institutes of Health (NIH) grant GM031575. SSB was additionally funded by the Instituto de Salud
Carlos III-Fondos FEDER (IFI14/00007) and a grant from Fundació Privada Daniel Bravo Andreu. SWA's work is additionally supported by $\mathrm{NIH}$ grant K01HL136700

\section{Availability of data and materials}

The data that support the findings of this study are available from the Genetic Analysis Workshop (GAW) but restrictions apply to the availability of these data, which were used under license for the current study. Qualified researchers may request these data directly from GAW.

\section{About this supplement}

This article has been published as part of BMC Genetics Volume 19 Supplement 1, 2018: Genetic Analysis Workshop 20: envisioning the future of statistical genetics by exploring methods for epigenetic and pharmacogenomic data. The full contents of the supplement are available online at https://bmcgenet.biomedcentral.com/articles/supplements/volume19-supplement-1.

\section{Authors' contributions}

$J A, R H, L L, K O, S S B$, and SWA formed the Causal Modeling Group at GAW2O and participated in discussions that led to this manuscript; JA drafted the section on defining causal inference and described the assumptions and details of his method; RH drafted Table 1 and the section on theoretical and practical challenges; $\mathrm{L}$ contributed to the description of their method; AJ drafted the context section and described the assumptions and details of her method; LL and SSB described analytical methods; SWA led the GAW20 Causal Modeling Working Group, and drafted and edited the manuscript. All authors read and approved the final manuscript.

Ethics approval and consent to participate

Not applicable.

Consent for publication

Not applicable.

\section{Competing interests}

The authors declare that they have no competing interests.

\section{Publisher's Note}

Springer Nature remains neutral with regard to jurisdictional claims in published maps and institutional affiliations.

\section{Author details}

${ }^{1}$ Department of Statistics, Columbia University, 1255 Amsterdam Ave, New York, NY 10027, USA. ${ }^{2}$ Institute of Genetic Medicine, Newcastle University, Central Parkway, Newcastle-upon-Tyne NE1 3BZ, UK. ${ }^{3}$ Department of Epidemiology, Biostatistics and Occupational Health, McGill University, 1020 Pine Avenue West, Montréal, Quebec H3A 1A2, Canada. ${ }^{4}$ Biomedical and Translational Informatics, Geisinger Health, 100 North Academy Ave, Danville, PA 17822, USA. ${ }^{5}$ State Key Laboratory of Genetic Engineering, Institute of Biostatistics, School of Life Sciences, Fudan University, 2005 Songhu Road Shanghai 200438, China. ${ }^{6}$ Département de Mathématiques, Université du Québec à Montréal, 2920 Chemin de la Tour, Montréal, Quebec H3T 1 J4, Canada. ${ }^{7}$ Cardiovascular Epidemiology and Genetics Research Group, IMIM (Hospital del Mar Medical Research Institute); Universitat Pompeu Fabra; CIBER Cardiovascular Diseases (CIBERCV), 08003 Barcelona, Catalonia, Spain. ${ }^{8}$ Department of Epidemiology, University of Alabama at Birmingham, 1665 University Blvd, RPHB 230J, Birmingham, AL 35294, USA.

Published: 17 September 2018

References

1. Didelez V, Sheehan N. Mendelian randomization as an instrumental variable approach to causal inference. Stat Methods Med Res. 2007:16:309-30.

2. Palmer C, Pe'er I. Statistical correction of the Winner's curse explains replication variability in quantitative trait genome-wide association studies. PLoS Genet. 2017;13:e1006916

3. Kraft P. Curses - winner's and otherwise - in genetic epidemiology. Epidemiology. 2008;19:649-51.

4. Day K, Waite LL, Alonso A, Irvin MR, Zhi D, Thibeault KS, Aslibekyan S, Hidalgo B, Borecki IB, Ordovas JM, et al. Heritable DNA methylation in CD4+ 
cells among complex families displays genetic and non-genetic effects. PLoS One. 2016;11:e0165488.

5. Feil R, Fraga MF. Epigenetics and the environment: emerging patterns and implications. Nat Rev Genet. 2012;13:97-109.

6. Bell JT, Pai AA, Pickrell JK, Gaffney DJ, Pique-Regi R, Degner JF. DNA methylation patterns associate with genetic and gene expression variation in HapMap cell lines. Genome Biol. 2011;12:R10.

7. Aslibekyan S, Almasy L, Province MA, Absher DM, Arnett DK. Data for GAW20: genome-wide DNA sequence variation and epigenome-wide DNA methylation before and after fenofibrate treatment in a family study of metabolic phenotypes. BMC Proc. 2018;12(Suppl 9). https://doi.org/10.1186/ s12919-018-0114-0.

8. Province MA. Methods for detecting methylation by SNP interaction in GAW20 simulation. BMC Proc. 2018;12(Suppl 9). https://doi.org/10.1186/ s12919-018-0140-y.

9. Howey RAJ, Cordell HJ. Application of Bayesian networks to GAW20 genetic and blood lipid data. BMC Proc. 2018;12(Suppl 9). https://doi.org/10.1186/ s12919-018-0116-y.

10. Jiang L, Zhao K, Klein K, Canty AJ, Oualkacha K, Greenwood CMT. Investigating potential causal relationships between SNPs, DNA methylation and HDL. BMC Proc. 2018;12(Suppl 9). https://doi.org/10.1186/s12919-018-0117-x

11. Li L, Wang C, Lu T, Lin S, Hu YQ. Indirect effect inference and application to GAW20 data. BMC Genet. 2018; 19(Suppl 1). https://doi.org/10.1186/s12863018-0638-3.

12. Sayols-Baixeras S, Tiwari HK, Aslibekyan SW. Disentangling associations between DNA methylation and blood lipids: a Mendelian randomization approach. BMC Proc. 2018; 12(Suppl 9)

13. Justice AE, Howard AG, Fernandez-Rhodes L, Graff M, Tao R, North KE: Direct and indirect genetic effects on triglycerides through omics and correlated phenotypes. BMC Proc 2018; 12(Suppl 9). https://doi.org/10.1186/s12919018-0118-9.

14. Rubin DB. Estimating causal effects of treatments in randomized and nonrandomized studies. J Educ Psychol. 1974;66:688.

15. Pearl J. Causal inference without counterfactuals: comment. J Am Stat Assoc. 2000:95:428-31.

16. Imbens GW, Rubin DB. Causal inference in statistics, social, and biomedical sciences. Cambridge: Cambridge University Press; 2015.

17. Morgan SL, Winship C. Counterfactuals and causal inference. Cambridge: Cambridge University Press; 2014

18. Bowden J, Davey Smith F, Burgess S. Mendelian randomization with invalid instruments: effect estimation and bias detection through egger regression. Int J Epidemiol. 2015;44:512-25.

19. Shoemaker R, Deng J, Wang W, Zhang K. Allele-specific methylation is prevalent and is contributed by $\mathrm{CpG}$-SNPs in the human genome. Genome Res. 2010;20:883-9.

20. Dekkers KF, van Iterson M, Slieker RC, Moed MH, Bonder MJ, van Galen M, Mei H, Zhernakova DV, van den Berg LH, Deelen J, et al. Blood lipids influence DNA methylation in circulating cells. Genome Biol. 2016;17:138.

21. Eu-Ahsunthornwattana J, Miller EN, Fakiola M. Wellcome Trust case control consortium 2, Jeronimo SM, Blackwell JM, Cordell HJ: comparison of methods to account for relatedness in genome-wide association studies with family-based data. PLoS Genet. 2014;10:e1004445.

22. Robins JM. Semantics of causal DAG models and the identification of direct and indirect effects. In: Green PJ, Hjort NL, Richardson S, editors. Highly structured stochastic systems. New York: Oxford University Press; 2003. p. 70-82.

23. Richardson TS, Robins JM. Single World Intervention Graphs (SWIGs): A Unification of the Counterfactual and Graphical Approaches to Causality. Working Paper 128. Center for Statistics and the Social Sciences. Seattle: University of Washington; 2013.

24. Ellis PJI, Morris TJ, Skinner BM, Sargent CA, Vickers MH, Gluckman PD, Gilmour S, Affara NA. Thrifty metabolic programming in rats is induced by both maternal undernutrition and postnatal leptin treatment, but masked in the presence of both: implications for models of developmental programming. BMC Genomics. 2014;15:49.

\section{Ready to submit your research? Choose BMC and benefit from:}

- fast, convenient online submission

- thorough peer review by experienced researchers in your field

- rapid publication on acceptance

- support for research data, including large and complex data types

- gold Open Access which fosters wider collaboration and increased citations

- maximum visibility for your research: over $100 \mathrm{M}$ website views per year

At BMC, research is always in progress.

Learn more biomedcentral.com/submissions 